\title{
Mathematical undecidability, quantum nonlocality, and the question of the existence of God
}

\author{
eds. Alfred Driessen and Antoine Suarez
}

\author{
Springer, 1997
}

This book offers a series of contributions written by scientists interested in a philosophical reflection on recent advances of science. Profound scientific theorems in modern mathematics and physics shed new light on two fundamental questions often only implicitly dealt with: is mathematical truth a purely man-made construction and is the physical reality behind the phenomena at least in principle always observable? The answers to both questions are closely related to the possible existence of an omniscient and omnipotent being. In this sense mathematical undecidability and quantum nonlocality are proposed as a possible road to metaphysical principles and eventually to God.

\section{Chapter XIV}

\section{The question of the existence of God in the book of Stephen Hawking: A brief history of time}

\author{
Alfred Driessen \\ Department of Applied Physics, University of Twente, P.O. Box 217, 7500 AE Enschede, The Netherlands (1997) \\ now (2012) Professor emeritus, Driessen.Alfred@gmail.com
}

\begin{abstract}
The continuing interest in the book of S. Hawking "A Brief History of Time" makes a philosophical evaluation of the content highly desirable. As will be shown, the genre of this work can be identified as a speciality in philosophy, namely the proof of the existence of God. In this study an attempt is given to unveil the philosophical concepts and steps that lead to the final conclusions, without discussing in detail the remarkable review of modern physical theories. In order to clarify these concepts, the classical Aristotelian-Thomistic proof of the existence of God is presented and compared with Hawking's approach. For his argumentation he uses a concept of causality, which in contrast to the classical philosophy neglects completely an ontological dependence and is reduced to only temporal aspects. On the basis of this temporal causality and modern physical theories and speculations, Hawking arrives at his conclusions about a very restricted role of a possible creator. It is shown, that neither from the philosophical nor the scientific view his conclusions about the existence of God are strictly convincing, a position Hawking himself seems to be aware of.
\end{abstract}




\section{Introduction}

In 1988 Stephen Hawking, a mathematician and physicist, published a book ${ }^{1}$ for the broader public, which soon after appearance became a best-seller. It was translated in more than 20 languages and, in parallel, a series of extended interviews were asked and given for important newspapers and magazines in many countries. Meanwhile more than 7 years have passed, and several studies have dealt with the physical ${ }^{2}$ and philosophical ${ }^{3},{ }^{4},{ }^{5}$ aspects treated in this book. In the following, a outline of the book and an analysis of the philosophical elements is given in the light of the metaphysics of Aristotle and Aquinas. The focus hereby is laid on the aspects relevant to the question of the existence of God.

In a first section a summary of the book will be presented based mainly on quotations of the book. The selections of the quotations of course are already a kind of comment, but in addition to this, explicit remarks are given which help to arrive to the conclusions of the present paper. In the following section the philosophical genre of this book will be identified: a speciality in the philosophical field, namely the proof of the existence of God. For comparison the view of the Aristotelian-Thomistic philosophical tradition will shortly be presented. Finally, in the last section, a discussion will be given, where the mutual relevance of the ideas of Hawking and the Aristotelian-Thomistic philosophy will be studied.

\section{Summary of the book}

The introduction by Carl Sagan already gives an important key for the understanding of the book. He writes6: This is also a book about God..... The word God fills these pages....Hawking is attempting ....to understand the mind of God....the conclusion of the effort...: a universe with no edge in space, no beginning or end in time, and nothing for a Creator to do. One clearly should have in mind, that besides presenting a popularisation of modern physical pictures about the universe, Hawking is entering the field of philosophy and eventually theology.

\footnotetext{
${ }^{1}$ S. Hawking, A brief history of time, from the big bang to black holes, Bantam Books, New York, 1988.

${ }^{2}$ M. Sachs, On Hawking's “A Brief History of Time” and the Present State of Physics, Brit. J. Phil. Sci. 44(3) (1993), pp 543-547.

${ }^{3}$ W.L. Craig, 'What Place, then, for a creator?': Hawking on God and Creation, Brit. J. Phil. Sci. (1990), pp 473-491.

${ }^{4}$ R.J. Deltete, Hawking on God and Creation, Zygon 28(4) (1993) pp 485-506.

5 A. Driessen, The question of the existence of God in the book of Stephen Hawking "A brief history of time", Acta Philosphica, 4, (1995), pp. 83-93.

${ }^{6}$ ref. 1, p. X.
} 
Hawking starts his book with a chapter called: Our picture of the universe. He gives a short historical description of the different pictures of the universe. About the beginning of the universe he says 7 : One argument for such a beginning was the feeling that it was necessary to have "First Cause" to explain the existence of the universe. He adds then immediately an explanation: Within the universe, you always explained one event as being caused by some earlier event. It is remarkable, that in this description of cause the timeaspect is essential, i.e. he neglects ontological causes, which are essential in the classical philosophy, and especially in metaphysics. On the basis of his definition of cause as working only from out the past he comes some two pages later to the first important conclusion about the role of a creator in a universe with a big bang8: An expanding universe does not preclude a creator, but it does place limits on when he might have carried out his job.

Interesting are his ideas about the fields of science, philosophy (metaphysics) and religion 9 : Some people feel that science should be concerned with only the first part (the laws that tell us how the universe changes with time); they regard the question of the initial situation as a matter for metaphysics or religion. For him metaphysics and religion seem to be quite close to each other, and distant to science. Hawking is ending the first chapter with some remarks about a complete unified theory and concludes ${ }^{10}$ : And our goal is nothing less than a complete description of the universe we live in. This remark gives rise to an important question: is a physicist able even with a perfect developed theory to give a complete description of the universe? What to say about the role of biology, medicine, sociology or even philosophy? Are they all included in physics?

In chapter 2 about Space and Time a history of science is given from the Greek up to the work of Penrose and Hawking which showed, that Einstein's general theory of relativity implied that the universe must have a beginning and, possibly, an end. ${ }^{11}$ After speaking in The Expanding Universe about the understanding of the universe from general relativity up to the state of knowledge in 1970, he points out the necessity of quantum mechanics for a next step in a deeper understanding. In chapter 4 The Uncertainty Principle he explains some basic principles of quantum mechanics, especially the uncertainty principle, which he shows to be essential to avoid that classical general relativity, by predicting points of infinite density, predicts its downfall. ${ }^{12}$ He remarks, that with the uncertainty principle a non-deterministic law in physics has been found. This has consequences also for the role of God, as scientific determinism ... infringed God's freedom to intervene in the world. ${ }^{13}$

In Elementary Particles and the Forces of Nature, chapter 5, he describes, starting from the Greek atomists the way to an overall theory of the four basic forces: gravitational,

\footnotetext{
${ }^{7}$ ref. 1, p. 7.

${ }^{8}$ ref. 1, p. 9.

${ }^{9}$ ref. 1, p.11.

${ }^{10}$ ref. 1, p. 13.

${ }^{11}$ ref. 1, p. 34.

${ }^{12}$ ref. 1 , p. 61

${ }^{13}$ ref. 1, p. 53.
} 
electromagnetic, weak nuclear and strong nuclear force. Up to now, there is only a partial result, the grand unified theory (GUT), including electromagnetic, weak nuclear forces and strong nuclear forces. Hawking comments ${ }^{14}$ : This title is rather an exaggeration: the resultant theories are not at all that grand, nor are they fully unified, as they do not include gravity.

Black Holes and Black Holes ain't so Black is treated in chapter 6 and 7. He first gives a historical overview, including the work of Penrose and himself, and shows, how general relativity gives rise to singularities, where the concept of space and time are seriously altered. A singularity, a concept taken from mathematical theories, denominates a special point or region in a function, where one has to divide by zero and where the function consequently is undefined. The functions used in the theory of general relativity can mathematically be considered as having a singularity, when they are applied to black holes. Later Hawking will speak about a second similar singularity, when he treats the big bang, the among physicists in general accepted starting point of the universe. For Hawking the concept of singularity is central in his reasoning. For within a singularity the known mathematical description of the physical reality breaks down, i.e. there is neither a deterministic nor a statistical description of the events of those regions. In this chapter Hawking is able to demonstrate quite convincingly, that the singularity in the center of a black hole can be circumvented, when one combines general relativity with the uncertainty principle. This seems to be the first combination of the two great theories of modern physics, general relativity and quantum mechanics, resulting in an unexpected and at a first sight paradoxical conclusion: black holes are not so black, i.e. they may emit energy or matter in form of radiation. Hawking considers this result as a glimpse of what a fully unified theory would bring in future. It is important to note that with this new approach, Hawking manages to get rid of the first class of singularities that are connected to black holes.

In the following chapter The Origin and Fate of the Universe Hawking tackles the problem of the second class of singularities, the big bang and eventually the big crunch. Unlike black holes, which are thought to be superabundant in the universe, the two species of the second class are unique; the big bang is considered as the starting point of the universe including time and all physical laws, the big crunch then is the final collapse with the end of time and the end of all known physical laws. After explaining in short the physical ideas connected to the big bang and big crunch, Hawking considers the philosophical implications of the big bang singularity: space-time would have a boundary a beginning at the big bang. ${ }^{15} \mathrm{He}$ then makes a statement about the laws of sciences in accordance with his restricted concept of causality, i.e. only temporary causality: These laws may have originally been decreed by God, but it appears that he has since left the universe to evolve according to them and does not now intervene in it. ${ }^{16}$ As one can see, only in the beginning, at the big bang singularity, a decisive role for God is possible.

\footnotetext{
${ }^{14}$ ref. 1, p. 74.

15 ref 1, p. 122.

${ }^{16}$ ref 1, p122.
} 
In the next pages the anthropic principle ${ }^{17}$ is introduced and different models of the development of the universe are presented. Hawking speculates about these models based on the general theory of relativity and quantum mechanics and ends with what he calls a proposal ${ }^{18}$ : space and time could be finite without boundary or singularity ${ }^{19}$, at least if one introduces the concept of imaginary time. Within his logic of the reduced concept of causality this proposal has profound implications for the role of God in the affairs of the universe. ${ }^{20}$ These implications, which are the central point of his book, have already been presented in the introduction by Sagan, and is worthwhile to quote once again: So long as the universe had a beginning, we could suppose it had a creator. But if the universe is really completely self-contained, having no boundary or edge, it would have neither beginning nor end: it would simple be. What place, then, for a creator? ${ }^{21}$ Some pages earlier Hawking already used an expression for the universe, it would just $B E^{22}$, which resembles the name of God in the Bible ${ }^{23}$ : Jahwe (I am who is). It should also be noted that Hawking in the development of his proposal is quite conscious of the speculative character of his argumentation: all statements, like the one just given, are expressed in terms of would, could, if, may, etc.

In The Arrow of Time Hawking considers the direction time passes, from past via the present to the future, this direction he calls the arrow of time. He considers three types of arrows: the thermodynamic arrow, related to entropy, i.e. the amount of disorder in a system, the psychological arrow, which relates to the human memory, as we only remember the past, and the cosmological arrow, which is the direction of time in which the universe is expanding. In the light of the "no boundary proposal" of the universe and the anthropic principle he shows the relation between the different arrows. His argumentation needs further philosophical study, because it is not clear whether the analogy between a computer memory and the human brain is strong enough to draw conclusions regarding the psychological arrow.

The Unification of Physics is the last chapter before the conclusion. Already the great aim of physics has been mentioned, the unification of the four basic forces in one single theory. But even with a complete unified theory, there are two reasons, why a physicist can't predict events in general: there is the uncertainty principle, where there is nothing we can do to get around that ${ }^{24}$. And there is another more practical inherent difficulty to solve

\footnotetext{
${ }^{17}$ The anthropic principle has been introduced by Hawking and B. Carter, and can be summarized: we see the universe the way it is because we exist.

${ }^{18}$ ref $1, \mathrm{p} 136$.

${ }^{19}$ There is a certain similarity with the Gödel universe, where the past and the future is a loop, see K. Gödel, Collected works, volume II, Publications 1938-1974, edited by S. Feferman et al., Oxford University Press, New York, 1990, pp. 189-216.

See also: G.C. Chaitin, Number and Randomness, algorithmic information theory - new results on the foundations of mathematics, this volume, chapter II

${ }^{20}$ ref. 1 p. 140.

${ }^{21}$ ref. 1 p. $140 \mathrm{f}$.

${ }^{22}$ ref. 1 p. 136

${ }^{23}$ Ex. 3,15

${ }^{24}$ ref. 1 p. 168.
} 
exactly the equations given by the theory. It is, e.g. not possible to solve exactly the motion of three bodies in Newton's theory of gravity. Being conscious of these fundamental restrictions Hawking nevertheless puts an aim quite ambitious for a physicist: our goal is a complete understanding of the events around us, and of our own existence ${ }^{25}$

The last chapter Conclusion summarises the way Hawking had led through the exciting area of modern physics. But now he draws conclusions, which are presented like different pieces of a mosaic, and which in general go far beyond physics into the realm of philosophy and eventually theology. About the situation before the theories of gravity and quantum mechanics are united, he writes: At the big bang and other singularities, all the laws would have broken down, so God would still have had complete freedom to choose what happened and how the universe began ${ }^{26}$. According to Hawking, however, with the new still not available unified theory and the no boundary proposal the situation would have changed largely: If the no boundary proposal is correct, he (God) had no freedom at all to choose initial conditions ${ }^{27}$.

After having made these statements, all in a conditional form, Hawking brings new pieces of thoughts into his mosaic of fundamental ideas regarding the universe, worthwhile to be quoted: Even if there is only one possible unified theory, it is just a set of rules and equations. What is it that breathes fire into the equations and makes a universe for them to describe? ${ }^{28}$ With this almost lyric sentence Hawking expresses what in the AristotelianThomistic metaphysics one could describe in terms of causa formalis and causa efficiens. The causa formalis is necessary, but not sufficient to cause the total effect. Besides this the causa efficiens is needed, who gives a set of ideas and 'formulas' an implementation in reality.

In the very same page Hawking invites the philosophers, the people who in contrast to scientists ask why instead of what the universe is, to keep up with the advance of scientific theories $^{29}$. He hopes that after the discovery of a complete theory a new area will come: Then we shall all, philosophers, scientists, and just ordinary people, be able to take part in the discussion of the question of why it is that we and the universe exist. If we find the answer to that, it would be the ultimate triumph of human reason-for then we would know the mind of God. ${ }^{30}$

\section{The central question: does God exist?}

From the foregoing it may be clear, that although the book is written by a physicist, it deals with a philosophical subject, a specialised theme of metaphysics, namely the proof of

\footnotetext{
${ }^{25}$ ref. 1 p 169.

${ }^{26}$ ref. 1. p 173.

${ }^{27}$ ref. 1, p.174.

${ }^{28}$ ref.1, p. 174.

${ }^{29}$ ref. 1, p. 174.

${ }^{30}$ ref. $1, \mathrm{p} 175$.
} 
the existence of God. Obviously the genre of the book is not affected by the positive or negative answer to the central question: "Does God exist?" Contemplating the two-three thousand years of history of philosophy from the ancient Greeks up to now, one observes a continuous interest in this central question. All the tools available to philosophers and scientists as, e.g. logic, metaphysics, history of philosophy and science itself, have been applied to clarify as much as possible the different aspects. Hawking as a scientist gives an important contribution to the scientific part of the question; regarding the philosophical aspects, he uses only a reduced selection of the knowledge until now obtained. The most comprehensive discussion of the proofs of the existence of God is probably given in the work of Aquinas, who resumed the different demonstrations in the famous five via's ${ }^{31}$. It is not the place here, to discuss in detail his argumentation, but a summary of the first way ${ }^{32}$, which Aquinas called the first and most obvious way, may give a proof of the strength of the philosophical argumentation.

\subsection{The first way of Thomas Aquinas}

In the first way Thomas uses ideas that already can be found with Plato ${ }^{33}$, Aristotle ${ }^{34}$ and Averoes. He starts from the common experience, that it is sure, that in this world some things move. Then he puts his first thesis: all what moves, is moved by some other. The proof of it is shortly given by an analysis of movement as being brought from being in potentia to being in actu, i.e. brought from being potentially in a certain state to being actually in that state. He comes to the conclusion: It is therefore impossible, that something in the same aspect and in the same way brings into movement as well is moved or moves itself. The next step in his argumentation is the thesis: If the mover himself is moving, then

\footnotetext{
${ }^{31}$ Thomas Aquinas, Summa Theologiae,I, q.2, a.3.

${ }^{32}$ For the interested reader an English translation of the first way is given below (from St Thomas Aquinas, Summa Theologiae, Latin text and English translation, Blackfriars 1964, Eyre\&Spottiswoode, London). In contrast to our quotations in the text, which follow closely the Latin of Aquinas, this translation uses more the concepts of today's English. The main difference is the translation of moveri, being moved, which is translated as being in process of change.

The first and most obvious way is based on change (ex parte motus). Some things in the world are certainly in process of change: this we plainly see. Now anything in process of change is being changed by something else. This is so because it is characteristic to things in process of change that they do not have the perfection towards which they move, though able to have it; whereas it is characteristic of something causing change to have that perfection already. For to cause change is to bring into being what was previously only able to be, and this can only be done by something that already is: thus fire, which is actually hot, causes wood, which is able to be hot, to become actually hot, and in this way causes changes in the wood. Now the same thing cannot at the same time be both actually $x$ and potentially $x$, though it can be actually $x$ and potentially $y$ : the actually hot cannot at the same time be potentially hot, though it can be potentially cold. Consequently, a thing in process of change cannot itself cause that same change; it cannot change itself. Of necessity therefore anything in process of change is being changed by something else. Moreover, this something else, if in process of change, is itself being changed by yet another thing; and this last by another. Now we must stop somewhere, otherwise there will be no first cause of the change, and, as a result, no subsequent causes. For it is only when acted upon by the first cause that the intermediate causes will produce the change: if the hand does not move the stick, the stick will not move anything else. Hence one is bound to arrive at some first cause of change not itself being changed by anything, and this is what everybody understands by God.

${ }^{33}$ Plato, Phaedrus

${ }^{34}$ Aristotle, Physica VIII
} 
he also has to be moved by some other, which in fact is a logical extension of the first thesis, and shows that there is a cascade of movers which in turn are moved by other movers. Aquinas now states, that there can be no infinite chain of movers and moved, as otherwise there would be no first mover, and consequently nothing, which could start the movement. His conclusion therefore is, that there must be a first mover, which is not moved by anything. And he ends his proof with: and this is what everybody understands by God.

About this first via some remarks should be given. Speaking about moving Aquinas considers all kind of changes, like getting hot, changing of color, change of position etc. In his second way, a similar proof is given, but then one should read instead of moved: caused by. It is of extreme importance to note that in the via's moved or caused by is always moved or caused by per se, i.e. if the mover or cause stops to move or cause, the effect also stops. With other words, the mover or the cause is acting in the present time. That means that also the cascade of movers and moved or causes and caused is completely in the present. The following example of a cascade or hierarchy of movers, which in a shortened way Aquinas already has mentioned in the explanation of the first way, may be a good illustration. It is the case of man, who is moving a ball along a certain trajectory, let say a circle: The ball is moved by a stick. The stick is moved by a hand. The hand is moved by a set of muscles. The muscles are moved by neural commands. The neural commands are moved by the brains. The brains are moved by the will, etc. The exact identification of the different levels in this cascade of movers may be a point of discussion, but one sees clearly that all movers are acting simultaneously and are acting per se, i.e. if one of the movers fails, there is no effect, in this case the ball would not follow the original trajectory.

The proof of Aquinas is quite subtle and looses its strength if one introduces even minor changes in the different steps. In the foregoing example, one could consider a ball shot by a soccer player, once the direct contact between shoe and ball is broken, the ball follows a trajectory that could be the intended one, but also could be drastically changed or even stopped by other movers or causes, like wind or a keeper's hand. In the case of movers as presented in this last example Aquinas would never conclude that there must be necessarily a finite cascade or a first mover.

Aquinas ends his proof with: and this is what everybody understands by God. One has to realise, that all of his reasoning is still in the field of philosophy and not theology. Only by starting from the daily experience of the movement of material things and logical thinking, he arrives at the necessity of something, which is the first mover or, in the second via, the first cause. Having obtained this result, it seems that he looks around to see, where to find this first mover. And the results of this exploration: the first mover is just that, what people in general understand by God. The first mover, a pure philosophical concept, can be identified with God, the central theme in theology. Notice however that Aquinas is not evoking Revelation to define God, but only the general understanding of people. In the Introduction ${ }^{35}$ an argument is given which may support the identification of the first mover with that people understand by God.

35 A. Driessen and A. Suarez, Introduction, this volume. 


\subsection{Hawking and the classical proof of the existence of God}

It is useful, to compare the different steps, Hawking is making in his attempt to clarify the question of the existence of God, with the classical proof of the Aristotelian-Thomistic philosophy, as has been presented just before in a summarised form. Hawking starts by using a changed concept of causality. We already quoted his explanation of the meaning of being caused, which for him is exclusively causality in time: Within the universe, you always explained one event as being caused by some earlier event... ${ }^{36}$. The exclusive use of this kind of temporal causality ${ }^{37}$, Aquinas explicitly excludes for his proof ${ }^{38}$. It may be clear that with the reduced concept of causality as used by Hawking, the original proof is strongly weakened.

Applying the temporal concept of causality, Hawking expects an intervention of a possible creator or God only in the beginning of the universe, as already has been shown by the quotations in section 2. As long as there is a beginning, which he identifies with the big bang singularity, there would be a role for a creator. If, however, the physical necessity of a beginning has been eliminated, the crucial question comes: What place then, for a creator? ${ }^{39}$. Hawking therefore comes in his main line of reasoning with the temporal concept of causality to the conclusion, that there is no logical need to assume the existence of a creator, as long as one only considers the universe of the physicists, which, as we have seen before, includes in his view the material world inclusive the human life. Nevertheless, he himself is convinced, that something is missing in his reasoning. Not only the question what, but also the question why should be asked: Why does the universe go to all the bother of existing? This question has not been answered yet, as up to now, most scientists have been too occupied with the development of new theories that describe what the universe is to ask the question why ${ }^{40}$.

\section{Discussion}

After having gone through the book of Hawking and presented the proof of the existence of God in the Aristotelian-Thomistic philosophy, one may want to look for the mutual implications, at least if there are any. Scientists, like philosophers, have their own working field, and the methods in science are quite different from those in philosophy. There is however an overlap: in the object, as scientists are dealing with the material reality as being material and philosophers with the same reality, the material and beyond that also with the

\footnotetext{
36 ref 1, p. 7.

37 This corresponds also to the Kantian view of causality (see A. Driessen and A. Suarez Introduction, this volume, and A. Suarez, Nonlocal phenomena, this volume, chapter X).

${ }^{38}$ L. Elders, De Metafysica van St. Thomas van Aquino in historisch perspectief, II: Filosofische godsleer, Uitgeverij Tabor, Brugge, 1987, p.150, see also Thomas Aquinas, In liber II Physicorum, lectio 6, n 195.

${ }^{39}$ ref. 1 , p. 141.

40 ref. 1 p. 174 .
} 
immaterial reality. And of course, always, the person, who is doing science, in other aspects is thinking as a philosopher, and often also the reverse is valid.

One may therefore say, there is an interaction between science and philosophy, and even between science and theology. Hawking himself gives an example, when introducing the Heisenberg uncertainty principle and discussing determinism: The doctrine of scientific determinism was strongly resisted by many people, who felt that it infringed God's freedom to intervene in the world, but it remained the standard assumption of science until the early years of the century. ${ }^{41}$ If that theory of total determinism in the physical world would have been proven to be true, then God's intervention in the material world would be bound to deterministic laws, and regarding human freedom, one could only consider at most pure internal decisions, which would not affect any physical reality.

If one now considers the main line of argumentation of Hawking, one is at first confronted with his restricted concept of temporal causality, which we have shown is contrary to the one used in classical philosophy. Nevertheless, even if one accepts this concept, his "proof" of non-necessity of a creator is not supported by physical evidence, but of ideas with a highly speculative character. He starts with theories, like the of relativity and quantum mechanics, which are shown to be valid by thousands of experimental verifications and which are accepted by practically all physicist. When discussing big bang, black holes, etc., there the scientific evidence is much weaker, and the ideas have a more hypothetical nature. Introducing, however, imaginary time and the no boundary proposal, Hawking himself is conscious of the speculative nature of his reasoning. One should be aware, if the scientist Hawking calls his ideas a proposal and admits that is far from being proven, then a philosopher (say Hawking or any other) may not use this argumentation as a decisive proof for the existence or non-existence of a creator. And if one reads the remarks of Hawking in his last chapter (see quotation, ref. 23), he seems to be aware of it.

There is one very interesting question left. The title of the book A brief history of time promises worthwhile and perhaps new ideas about time. A widely discussed question in philosophy is, whether the universe is eternal, and - this is not the same question - whether the universe is created. Science was not able to give an answer. With the introduction of the big bang hypothesis, based on the work of Penrose and Hawking, many considered this as the proof, that there was a beginning and therefore a creation. With the no-boundary proposal Hawking has not proven, that the universe is eternal, simple being. What he has shown, is that for a scientist at the top of the knowledge about the universe, the older standard big bang hypothesis is not necessarily true, and that the idea of a universe without beginning can not be rejected on purely scientific reasons. It is therefore still a matter of discussion. Coming back to Aquinas, one finds the problem of creation of the universe in time or creation from eternity ${ }^{42}$. His conclusion is, that it is possible to demonstrate the ontological dependence of the universe from God, but not the beginning in time. Only

\footnotetext{
${ }^{41}$ ref. 1 p. 53.

${ }^{42}$ for a discussion, see, e.g. L.J. Elders, De natuurfilosofie van Sint Thomas van Aquino, Uitgeverij Tabor, Brugge, 1990, p. $138 \mathrm{ff}$.
} 
additional information, as is given in theology by revelation, could give an answer ${ }^{43}$. For Aquinas evidently the answer to this question is not relevant for the demonstration of his 5 via's. This has an enormous impact on the philosophical value of the input of science as has been delivered by Hawking. The main line of his reasoning does not affect the philosophical proof of the existence or non-existence of a creator, at least in the philosophy of Aquinas. What then is the value? Not a small one, one may say, namely bringing people to think and stimulate them to ask why.

${ }^{43}$ In the Jewish-Christian tradition this information is found in Gen. 1.1: In the beginning..... 